\title{
Sensaciones corporales y el Trabajo Fin de Grado: una propuesta práctica
}

\section{Bodily sensations and the Final Degree Project: A practical proposal}

\section{Eider Gamboa Ruiz de Eguilaz¹, Asier Huegun Burgos² \& Pello Jauregi Etxaniz ${ }^{3}$}

Fecha de recepción: 02/03/2021; Fecha de revisión: 28/09/2021; Fecha de aceptación: 05/10/2021

Cómo citar este artículo:

Gamboa, E., Huegun, A. \& Jauregi, P. (2021). ¿Pueden las sensaciones corporales ser parte del escenario del desarrollo del Trabajo Fin de Grado? Una propuesta desde el Aprendizaje Basado en la Indagación. Revista de Innovación y Buenas Prácticas Docentes, 10(2), 27-39.

Autor de Correspondencia: eider.gamboa@ehu.eus

\section{Resumen:}

En este artículo se expone la investigación realizada para construir nuevos espacios de pensamiento a partir de sensaciones corporales en el proceso de elaboración del Trabajo Fin de Grado en educación superior. A partir del Aprendizaje Basado en la Indagación, se han desarrollado ejercicios basados en el enfoque corporal para profundizar en este tipo de aprendizaje. Las sensaciones que emanan de las propias vivencias son fuente de conocimiento en los procesos de reflexión y acción que llevan a cabo los estudiantes de la educación universitaria. A través de herramientas cualitativas para la recolección de datos se han obtenido resultados significativos en cuanto a la relevancia de la propia experiencia de los estudiantes, así como la función de las sensaciones corporales en los procesos reflexivos.

Palabras clave: innovación pedagógica, intuición, trabajos dirigidos, universidad, indagación.

\section{Abstract:}

This article presents the research carried out to build new spaces of thought from body sensations in the process of creating the Final Degree Project in Higher Education. For this purpose, based on Inquiry Based Learning, it has developed exercises based on the body approach to deepen this type of learning. The sensations that emanate from one's own experiences are a source of knowledge in the reflective and action processes carried out by students in university education. Through qualitative tools for data collection, we have obtained significant results in terms of the relevance of students' own experience, as well as the function of bodily sensations in the reflective processes.

Key Words: directed exercises, educational innovation, intuition, university, inquiry.

\footnotetext{
' Universidad del País Vasco/Euskal Herriko Unibertsitatea (UPV/EHU) (España), eider.gamboa@ehu.eus; CÓDIGO ORCID: https://orcid.org/0000-0002-8206-0941

2 Universidad del País Vasco/Euskal Herriko Unibertsitatea (España), asier.huegun@ehu.eus; CÓDIGO ORCID: https://orcid.org/0000-0003-0703-0766

3 Universidad del País Vasco/Euskal Herriko Unibertsitatea (España), pello.jauregi@ehu.eus; CÓDIGO ORCID: https://orcid.org/0000-0002-8806-4759
} 


\section{INTRODUCCIÓN}

La idea fundamental de la investigación que se presenta a continuación es la de ayudar en la construcción de nuevos espacios de pensamiento partiendo de las sensaciones corporales que se tienen respecto a las propias experiencias como fuente de conocimiento en los procesos reflexivos y de acción dentro del proceso de creación del Trabajo Fin de Grado (TFG). La investigación se ha llevado a cabo con los estudiantes universitarios de cuarto curso de los grados de Educación Social y Pedagogía de la Universidad del País Vasco/Euskal Herriko Unibertsitatea (UPV/EHU) bajo la convocatoria de Proyectos de Innovación Educativa (PIE) 2018-2019 de dicha universidad, habiendo sido implementada entre septiembre del 2018 a junio de 2020.

EI TFG culmina el proceso formativo universitario de los grados y nos ofrece un espacio privilegiado de creación desde donde surge una inquietud para diseñar proyectos innovadores dentro de una temática relevante del campo de la educación (Ferrer, Carmona, \& Soria, 2008):

Y es que para poder avanzar en el TFG es necesario que toda acción tenga un sentido para nosotros, dado que es ahí donde surge la motivación, el ansia de saber, buscar nuevos conocimientos, seguir prosperando y evolucionando; en definitiva, es cuando emerge el verdadero aprendizaje (p.98).

La UPV/EHU siendo consciente de la necesidad de generar procesos de cambio e innovación docente en los escenarios de enseñanza-aprendizaje, promueve el modelo IKD (Ikaskuntza Dinamikoa eta Kooperatiboa/Aprendizaje Dinámico y Cooperativo) que pone énfasis en que el alumnado sea dueño de su aprendizaje, siendo formados de forma integral, flexible y adaptada a las necesidades de la sociedad (UPV/EHU, 2019). Esa idea va en la misma línea que la que defiende Esteves (2018) haciendo hincapié en el carácter innovador de ciertas prácticas pedagógicas, como la introducción de nuevas pedagogías y nuevas prácticas en el quehacer de los docentes en los procesos de enseñanza-aprendizaje.

Todo ello nos lleva a poner el foco en la visión educativa que defiende que en los procesos de enseñanza-aprendizaje la parte intuitiva y sensorial también tiene una función primordial:

Transformative learning does this to an unusual degree. It engages and involves the whole person, and affects change in deep levels of values and belief through a process of re-perception and re-cognition. It is not the just a matter of intellectual or conceptual learning, but engages our emotional and intuitive selves as well" (Sterling, 2004, p.56).

Creemos que dicha visión viene acompañada de los nuevos modos de entender la universidad que se están gestando a lo largo y ancho del mundo universitario. Queremos destacar la visión del profesor del MIT Scharmer (2020) que busca en su decálogo de la nueva universidad apoyar el desarrollo de la alfabetización vertical. Entender la universidad como un entorno de aprendizaje en el que el alumno pueda dar un paso hacia su potencial futuro más alto en el contexto de desafíos sociales prácticos. El contexto actual requiere, entre otras, la mejora de la forma en que prestamos atención y escuchamos, la forma en que conversamos, dialogamos y pensamos, para mejorar la forma en que nos organizamos y coordinamos. Entre las propuestas que realiza queremos destacar el embodiment como parte de ese decálogo que invita al cambio de nuestra manera de afrontar los procesos de enseñanzaaprendizaje: 
Move the inner place of learning from the head to the heart and from the heart to the hand. The essence of learning in this century revolves around activating the intelligence of the heart and then putting it to use in serving the needs of others and the whole. (Scharmer, 2020, p.7)

Desde el contexto de los desafíos sociales prácticos emanan las inquietudes respecto a una situación socioeducativa para llegar a problematizarla con el fin de transformarla (Dewey, 2004; Freire, 2008) y para ello, el Aprendizaje Basado en la Indagación -Inquiry Based Learning IBL- (Peñaherrera, Chiluiza \& Ortiz, 2014) nos ofrece la oportunidad de que el alumnado cree sus propias preguntas, indague para buscar las respuestas y éstas sean nuevas e innovadoras. Construir puentes entre la experiencia del alumnado (prácticas, experiencias educativas y vitales,...) y el espacio simbólico (palabras, teorías, discursos, reflexión) nos lleva a tener en cuenta las sensaciones corporales que surgen de las experiencias: se entrelazan e influyen entre sí para crear conocimiento. Los procesos de indagación ayudan a descifrar el conocimiento que hay en las experiencias (Aramendi, Arburua \& Buján, 2018), es decir, crear campos experienciales (Dewey, 2004) y abrir procesos de indagación desde ahí.

La característica fundamental de las experiencias es todo ese entramado vivenciado que el alumnado todavía no sabe ponerlo en palabras, algo así como un saber borroso y confuso (Barceló, 2015). Es una fuente fértil para crear procesos de indagación, reflexión, praxis e interrogantes que puedan dar inicio al TFG y a su posterior desarrollo. Las sensaciones corporales que experimentamos, aunque sean confusas, y al principio débiles, contienen innumerable información acerca de nuestra experiencia (Barceló, 2015; Gendlin, 2009; Tokumaru, 2011).

Tal y como señala Jordi (2011):

Evidence from neuroscience points us toward the physiology of mind-body integration. However, it also shows that we have a significant structural and functional tendency toward non-integration and dissociation of thought from embodied experience. Disconnection of mind from body, like mind-body integration, is a physiological proclivity not just an ideological construct. Biology provides us with the capacities, and we make the choices, develop the inclinations, and harden the patterns psychologically, socially, and culturally (p.183).

Indagar es descifrar el sentir que surge desde la experiencia (Zambrano, 1996). Cuando hablamos de la experiencia nos referimos a que a menudo se experimentan significados sentidos fuera de la simbolización verbal, sin definir, ni se acierta a ponerles palabras; en su lugar se tiene una percepción, o alguna palabra que no representa nada ("esto" o "algo"). Cuando este es el caso, el significado permanece implícito, no reconocido explícitamente. La sensación al principio puede que resulte vaga: no sabemos qué decir o cómo caracterizarla, pero notamos una cualidad única. El experienciar contiene una significación, no es sólo la sensación interna de nuestro cuerpo. No es sólo el mero hecho de sentir algo. La mayoría de las veces la sensación que se atiende en el interior en relación a un asunto de la vida o profesional resulta confusa al principio. Incluso puede resultar vaga o no discriminable (Barceló, 2015), pero se siente el cuerpo de manera diferente. Una característica de esta sensación es que se experimenta como un todo complejo que abarca muchas ramificaciones (Barceló, 2015; Gendlin, 1983, 1997). 
Por lo tanto, (la sensación) "es como una multiplicidad de aspectos que se manifiestan en una sola sensación. Esta sensación constituye un dato significativo de nuestra experiencia y hay que dejarla venir porque surge novedosamente cuando atendemos el centro de nuestro cuerpo" (Barceló, 2015, p.171).

\begin{abstract}
Además:
La prioridad de la experiencia, que es una experiencia vital, se antepone entonces al lenguaje porque surge de las vivencias originarias que se producen, por lo que nos afecta de nuestro estar en el mundo en forma de impresiones, emociones, sentimientos y sensaciones que sentimos corporalmente. Pero la experiencia es siempre experiencia significada y la significación la otorga el lenguaje (...) Todo este cúmulo fenoménico produce en la persona material de la experiencia, sensaciones que pueden atenderse y adquirir significado. Este flujo líquido que cambia a cada instante y ondea en el campo existencial de cada individuo configura la experiencia de este individuo como ser en su contexto (...) A todo este marco nos referimos con el término experiencia. La experiencia conforma esta «sensación de...» producida por algún conglomerado de estímulos que nos afectan y acontece en función de las interacciones que establecemos con el medio y con los demás. Lo que sentimos nos acontece" (Barceló, 2015, p. 5).
\end{abstract}

En esa dirección podemos afirmar que los acontecimientos afectan a un sujeto que las vive de determinada manera en determinado momento; el experienciar (Gendlin, 1997), por lo tanto, es un fenómeno subjetivo e interaccional. De ahí que poner en valor las sensaciones corporales nos da la oportunidad de rescatar datos significativos de nuestra experiencia. Descifrar el sentir es enlazar y conectar lo experiencial con lo simbólico, que encaje la palabra con la sensación que se está sintiendo.

Ese saber borroso y difuso es esa sensación corporal que transita entre la certeza y la incertidumbre y es generadora de conocimiento y el punto de partida para crear procesos de indagación. Se trata de experimentar una relación entre dos estados: por un lado, la incertidumbre resuelta; por otro, la incertidumbre que queda por resolver (Wagensberg, 2008). Los procesos de indagación dan la oportunidad de situar al alumnado en lo que Zambrano (1996) llamaba la actitud filosófica y la actitud poética: la primera se refiere a hacer(se) preguntas y formular las inquietudes que se tienen respecto a una situación o tema, viene a generar caos y desorden; y la segunda actitud viene a ordenar el caos, es la respuesta, la calma y en la que una vez descifrada se encuentra el sentido a la indagación.

La experiencia es todo ese material disponible, aún no simbolizado pero sí palpable como una presencia interna sentida, como una sensación que definimos provisionalmente con la palabra algo (Barceló, 2015). Todo eso está compuesto, seguramente, por hechos y experiencias del devenir diario que son, como diría Gadamer (1977), moldeadas por los esquemas culturales y psicológicos, y que hacen de estar en el mundo una manera de ser en el mundo. Para ello, tenemos hay que situarse en esa sensación de frontera, para prestarle atención un tiempo a algo que es sentido, pero que es poco claro por el momento y que es desde donde se pueden crear procesos de indagación.

Para una mejor comprensión de dicho fenómeno encontramos el concepto de lógica difusa (fuzzy logic) propuesto desde el campo de la física cuántica. Como nos indica Velarde (1996): 
La innegable realidad de lo difuso en los procesos del pensamiento y del razonamiento humano sugiere que buena parte de la lógica propia del razonamiento humano no es la lógica clásica bivalente o incluso polivalente, sino una lógica con cuantificadores difusos, con vectores difusos, con valores de verdad difusos y con reglas de inferencia difusas" (p.436).

La cualidad borrosa y difusa de las sensaciones es una cualidad propia en sí misma. El propio creador de la lógica difusa lo explica bien claro al afirmar que la lógica difusa es una salida radical de los sistemas lógicos tradicionales y que es mucho más que un sistema lógico (Zadeh, 2014). La lógica difusa tiene como objetivo proporcionar una base para la formalización de dos capacidades humanas notables. Primero, la capacidad de comunicar, razonar y tomar decisiones racionales en un ambiente de imprecisión, incertidumbre y parcialidad de la verdad. En segundo lugar, la capacidad de realizar una amplia variedad de tareas como conducir un automóvil en el tráfico urbano pesado, sin mediciones ni cálculos. Es por ello, que la lógica difusa está diseñada para tratar la información imperfecta:

Fuzzy logic is designed to deal with imperfect information. Imperfect information is information which in one or more respects is imprecise, uncertain, incomplete, unreliable, vague or partially true. In the real world, such information is the norm rather than exception (Zadeh, 2014, p. 2).

Las ideas de Prigogine (2008) sobre la generación del orden desde el caos se encuentran en la misma línea: la inestabilidad es el punto de partida para nuevas formulaciones o leyes físicas. Por lo tanto, también desde el desorden y el caos se puede surgir un nuevo conocimiento, una idea, una nueva percepción respecto a un hecho, o, una línea de acción en el campo socioeducativo.

Siguiendo a Gromi (2013), se puede afirmar que para el alumnado de Educación Social y Pedagogía el aprendizaje debe de cumplir tres premisas las cuales se pueden acompañar del IBL para su implementación y desarrollo:

- que permita a los estudiantes apropiarse de los modos de conocer,

- que impulse a los estudiantes a que interroguen su experiencia y, desde allí, construyan significado (es decir, la simbolicen, por ejemplo, en un discurso teórico), y

- que ayude a los estudiantes a tratar la información y organizarla en sistemas de conocimientos y representaciones eficaces de la realidad.

\section{DESARROLLO DE LA EXPERIENCIA DE INNOVACIÓN}

\subsection{Objetivos}

La investigación ha tenido por objetivo analizar la percepción individual del alumnado participante en el proyecto SentiGRAL "Construyendo nuevos espacios de pensamiento desde las sensaciones corporal en el proceso de creación de los TFG", respecto al diseño e implementación de las herramientas didácticas desarrolladas con el fin de crear conocimiento desde sus propias experiencias. 


\subsection{Participantes}

En total han participado en el estudio 24 alumnos y alumnas de cuarto curso de los grados de Educación Social y Pedagogía a lo largo de los cursos 2018/2019, 2018/2019 y $2019 / 2020$. Ninguno de ellos contaba con experiencia previa en el enfoque corporal y aceptaron libremente participar en el proyecto, disponiendo en todo momento de la posibilidad de cambiar en el modo de tutorización del TFG a la opción metodológica tradicional.

\subsection{Herramientas}

El proyecto se articula sobre tres herramientas que se presentan a continuación:

a) TAE (Thinking at the Edge, Pensando desde el Borde) [Gendlin (1983, 1997, 2009); Tokumaru (2011); Krycka (2015)].

EI TAE tiene por objeto buscar una forma de pensamiento basada en la vivencia corporal que surge de las ideas. Brinda un camino para dejar que irrumpan nuevas y variadas formas de lenguaje espontáneo, para diferenciar significados desde las sensaciones, para facilitar que aparezcan estos significados en múltiples direcciones y para posibilitar que surjan nuevas relaciones de conceptos provenientes de las sensaciones significativas que experimentamos (Aguilar, 2011; Barceló, 2015; Tokumaru, 2011). Thinking at the Edge es un término que significa pensar innovadoramente y tiene por objetivo llegar hasta el "borde" (edge), hasta lo ya desarrollado en un campo, hasta donde ya sabe el alumno, para a partir de ahí poder innovar (Alemany \& Aguilar, 2019). Elegimos TAE, por lo tanto, para poder atender a esa sensación corporal y atenderla con nuestra reflexión. En síntesis: para darle un significado personal desde las vivencias propias a las inquietudes que tiene el alumnado en el proceso de indagación e intenta resolver.

Como apuntan Nagamine, Fujieda, e lida (2018) we postulated that we would be able to help pre-service teachers effectively explore emotional, as well as cognitive, aspects of teaching practices, and verbalize practical knowledge through the meaningconstruction process of a felt sense (p.151). manera:

Los pasos de los que consta el proceso se resumen brevemente de la siguiente

- Preparar el cuerpo, elegir un campo teórico o experiencia sobre la que trabajar. Dejar que se forme una sensación sobre ese tema o experiencia.

- Expresar esa sensación, escribir varias líneas desde esa sensación de un modo desordenado e ilógico.

- Expandir, ver a dónde llevan los conceptos expresados o escritos.

- Entrecruzar, cruzar entre sí conceptos e ideas que, a primera vista, no tiene nada en común, para que, desde ahí surjan ideas nuevas, tanto teóricas como prácticas.

- Desplegar, dar forma a lo realizado hasta ese momento: escribir los apartados del trabajo o realizar las acciones necesarias para desarrollarlo.

- - Comprobar, leer o escuchar lo escrito hasta ese momento del proceso y ver cómo resuena en el cuerpo para realizar cambios o modificaciones oportunas.

- Recibir y proyectar, preguntarse y recoger las sugerencias propias para futuras indagaciones.

b) Focusing o enfoque corporal (Alemany, 2007; Gendlin, 1983, 1997, 2009) 
El Focusing, el enfoque corporal de los problemas o situaciones, pretende atender a las repercusiones que un asunto tiene en el cuerpo. Ese sentir corporal es esencial en la forma de asimilar el tema enfocado, que además pude ser generador de conocimiento y cambio (Gendlin, 2009).

c) Una guía didáctica creada ad hoc para la elaboración del TFG.

En la guía didáctica SentiGrAL(nombre dado a la guía para diferenciarla de la guía tradicional del TFG) se concretan al detalle los pasos y tareas a realizar por parte del alumnado para el desarrollo del TFG. El proceso consta de tres fases, divididas en 15 subtareas en la que se integran ejercicios tanto de TAE como de Focusing.

Para la validación de la guía se ha realizado una triangulación de expertos en la que han participado dos docentes universitarios con amplia experiencia en la tutorización de los trabajos fin de grado. Concretamente, uno de ellos es experto en procesos de enfoque corporal, y el otro experto en procesos de formación en la universidad. Con la elección de estos perfiles se ha pretendido analizar la viabilidad de la aplicación de esta guía para el tutor/a que no cuente con una formación propia en el enfoque corporal. Tras el análisis de las entrevistas realizadas se han recogido sus sugerencias y mejoras para incorporarlas en la guía final.

\subsection{Fases}

El diseño realizado para desarrollar el TFG consta de las siguientes fases: 1) Explorar en las experiencias para formular la pregunta estructurante; 2) Desarrollo del marco teórico y/o diseño e implementación de la intervención; y 3) Creación de los resultados y discusión. Se han llevado a cabo sesiones tutoriales individuales (de 6 a 8 por cada alumno/a).

1) Explorar en las experiencias para formular la pregunta estructurante.

Mediante la utilización de TAE y Focusing se ahonda en esa sensación que trae el/la alumno/a respecto a una temática que tiene que ver con alguna vivencia suya. El significado permanece implícito y mediante las dos herramientas mencionadas se intenta poner la atención a eso que hay de "novedoso" en la vivencia. Al final de esta fase el/la alumno/a encuentra su pregunta estructuradora que hace que, a modo de vector, el trabajo adquiera dirección e intensidad. Desde la sensación emergen ideas, preguntas, palabras... es la fase de despliegue, para después, seguir con la fase de cierre en la que el/la alumno/a elige, desde la sensación, dos ideas o palabras de todo el abanico que ha creado. Busca nuevos enfoques y explora las posibilidades de todos ellos para, después, elegir mediante la sensación corporal cuál de todo eso encaja hasta que logra sentir la pregunta estructurante como suya. En esta fase se han dedicado dos o tres sesiones con cada alumno/a.

2) Desarrollo del marco teórico y/o diseño e implementación d la intervención.

El/la alumno/a empieza a desplegar el trabajo. Aquí se abren dos caminos: por un lado, puede comenzar a construir el marco teórico y, por otro, empezar a diseñar e implementar (metodología e intervención) aquello que va a llevar a cabo para responder a la pregunta-estructurante, bien sea un estudio bibliográfico, un diseño de intervención en un barrio, una intervención en un recurso educativo en medio abierto... Al mismo tiempo, en esta fase se definen los objetivos y los instrumentos metodológicos que va a 
utilizar: cuestionario, entrevistas, estudio etnográfico... El esquema expansión-cierre es la base de esta segunda fase. La relación sensación-símbolo es lo que hace de guía a la hora de elegir las ideas fundamentales del marco teórico, los objetivos, las actuaciones del diseño, las acciones y la recogida de datos. Esta fase ha tenido tres sesiones con el alumnado.

3) Creación de los resultados y discusión.

En esta fase la atención ha estado puesta en los resultados y conclusiones que ha obtenido el/la alumno/a después de su trabajo y en la discusión de los mismos. Para la creación de los resultados se ha partido de los objetivos de cada TFG. Se ha basado, sobre todo, en ejercicios de focusing que se recogen en la guía. En las conclusiones se les ha pedido que respondan a la inquietud que ha sido el germen del TFG. A esta fase se le ha dedicado entre una y tres sesiones de tutoría.

\subsection{Procedimiento de recogida de datos}

Se han utilizado los siguientes instrumentos para la recogida de datos: a) entrevista en profundidad al final del proceso con cada alumno/a; b) diario de seguimiento del docente; y c) sesiones de trabajo del grupo de investigación.

\subsection{Análisis de datos}

El análisis de la información recogida ha seguido el siguiente proceso (realizada con el soporte informático Nvivo): a) reducción de la información, b) organización y presentación de la información y c) análisis e interpretación de los resultados (Miles \& Huberman, 1994).

El sistema categorial ha sido emergente, consistiendo en ir diferenciando unidades de significado a los que se les ha asignado una categoría, la cual se ha ido concretando según su contenido. Los testimonios de los y las participantes han marcado la conformación de una categoría, teniendo en cuenta que en las entrevistas realizadas se han planteado preguntas tras una primera revisión bibliográfica y haciendo referencia a los objetivos de la investigación. Por lo tanto, como afirman Lukas y Santiago (2009), en este tipo de estudios, el sistema categorial surge del análisis y desarrollo de toda esa información.

Tabla 1.

Sistema categorial.

\begin{tabular}{ll}
\hline \multicolumn{1}{c}{ Categoría } & \multicolumn{1}{c}{ Subcategoría } \\
\hline Indagar desde las sensaciones & Valorar la experiencia previa del alumnado \\
& $\begin{array}{l}\text { Comenzar del proceso desde las } \\
\text { sensaciones }\end{array}$ \\
& $\begin{array}{l}\text { Indagación en la experiencia y personalizar el } \\
\text { trabajo }\end{array}$ \\
\hline Herramientas didácticas & $\begin{array}{l}\text { Ejercicios TAE y Focusing } \\
\text { Percepción del alumnado respecto al } \\
\text { proceso }\end{array}$ \\
& $\begin{array}{l}\text { Aparición de ideas significativamente } \\
\text { sentidas }\end{array}$ \\
& Reflexionar desde el sentir \\
\hline
\end{tabular}

Fuente: Elaboración propia 


\section{RESULTADOS}

Para la elaboración de los resultados nos hemos basado en las tres categorías y en sus correspondientes subcategorías.

\subsection{Sentir las experiencias abre un proceso de indagación creativo}

Emerger y dar forma a las inquietudes del alumnado que surgen de las vivencias propias hace que problematicen un tema del ámbito educativo, lo hagan suyo e inicien un proceso de indagación creativo. Los estudiantes han creado interrogantes a partir de su experiencia y la sensación interna ha hecho de guía del proceso para discernir lo que sí es válido para su TFG y lo que no: "Indagar es ayudar a definir la idea, es decir, la pregunta que intentaba responder. La pregunta me dirigía" (A4). El proceso ha hecho que el alumnado personalice el TFG y el proceso de indagación en el cual mirar(se) y en el que las sensaciones ayudan a desplegarlo: "He conseguido hacer una conexión con todo... el mirar cómo estás tú: sensaciones, ideas... partir de unas preguntas para mirar la sensación y hacer conexiones con la teoría e información para profundizarlo. A mi me ha valido" (A1).

Prestar atención a todo ese bagaje experiencial que trae el alumnado al inicio del TFG, y darle status de conocimiento ha generado una sensación "de algo nuevo": "La importancia que tiene la experiencia, que la inquietud para hacer el TFG nazca desde las tripas. Al final la experiencia me da herramientas para empezar algo. La experiencia te da la base. El conocimiento teórico en sí mismo no te hace hábil en la práctica. Tienen que ir de la mano" (A2). "Este proceso le da un valor añadido al TFG porque ya no solamente haces un trabajo que te guste, sino ¿Qué puedo aportar yo con él?... y a mí eso como Educadora Social es fundamental. Tenemos una responsabilidad $y$ te presentas al mundo de otra manera" (A13).

A su vez, todo eso sentir ha supuesto que el alumnado personalice su TFG y lo identifique como suyo: "Lo de las tripas y el sentir me ha ayudado. Es una fase muy importante de indagación... sacar toda esa inquietud que tienes dentro es la base, eso es hacer el trabajo, el sentirlo. Al final es como algo que sientes ahí: en mi caso parte de mi experiencia laboral" (A4). "Realizar la pregunta me ha ayudado para enfocar mi tema. A personalizar mi tema. Si no haces eso creo que se queda todo como en el aire" (A5).

\section{Las herramientas TAE y Focusing han ayudado a activar el proceso de indagación.}

Las tres herramientas (TAE, Focusing y la guía) han ayudado en el proceso de indagación en tres direcciones: carácter vectorial del trabajo, crear redes conectivas entre el sentir y la teoría, y reflexionar desde el sentir:

La pregunta estructurante ha ayudado a afinar el proceso al encajar la sensación y la palabra (símbolo). Todo eso hace que tome carácter vectorial: una clara dirección (necesidad de responder a la pregunta) e intensidad (el alumno siente que el aprendizaje vendrá al responder a la pregunta). "A mí tener clara qué pregunta debería de responder me daba fuerza, me hacía sentir con claridad dónde debía de buscar el marco teórico, me guiaba por esa sensación" (A12). Desde la sensación que emerge de la experiencia (desde el conocimiento implícito) han realizado un recorrido propio en el que el tratar de responder a la pregunta-estructurante han dado sentido al proceso. "A los educadores 
nos cuesta mucho darle nombre a lo que hacemos y en este proceso me ha ayudado el resonar en el cuerpo lo que ponía por escrito" (A2).

Afinar con la pregunta que surge de la experiencia y ponerla en palabras es clave: "La pregunta es mía (...) Me ha servido para discriminar la información que era significativa para mí y cuál no, la que era válida. Gracias a la primera fase (crear la pregunta) sabía qué información quería buscar" (A5). "Las ordeno en base... mi pregunta. Tener, sentir claramente cuál ere la cuestión que quería resolver en el TFG" (A4).

Abrir nuevos campos de reflexión desde el sentir. Los ejercicios de despliegue y cierre han traído un fluir entre lo personal y lo teórico, donde se han entrelazado aspectos importantes y significativos del TFG con sus propias reflexiones, ideas y líneas de acción: "Trabajar así es un poco a la inversa: partimos de nuestra experiencia y luego lo sustentamos con teoría, autores (...) Creo que así se genera nuevo conocimiento. Algo nuevo (...) "El proceso de abrir y cerrar desde la sensación a mí me abrió los ojos" (A2). "Indagar, indagar... ha sido el faro del trabajo, yo buscaba una respuesta a una pregunta que surgía de mis vivencias personales y profesionales, necesitaba responder a la pregunta, en mi caso hacer una propuesta de intervención" (A21).

Han contribuido a crear puntos de vista nuevos: "Lo que me salía de dentro, estuviera bien o mal yo lo apuntaba y luego me ayudaba a elegir. Eso ha sido muy intenso, me han salido cosas que no pensaba que me iban a salir. A se ejercicio (expansión/cierre) le puse el nombre de "patinaje" (A4). Al fin y al cabo, la idea de patinar, creada por una alumna es una metáfora muy adecuada para describir el proceso de indagación y los instrumentos que hemos utilizado: al patinar, divagamos de un lado para otro deslizándonos, buscando un lugar en el que encaje eso que estoy buscando, en nuestro caso una idea, una acción, una propuesta... En las siguientes palabras queda muy bien reflejado: "De una idea me ha llevado a otra idea y de ahí a otra y de ahí, a la final, a la que sentía que era la válida. Las ideas que al principio no parecían que tenían relación... ves que se relacionan" (A4).

En el diario recogimos también datos en la misma dirección: "La fase de expansión/ cierre es muy fructífera, se crean espacios novedosos, nuevos, ideas, lógicas, no lógicas y el alumno/a no las enjuicia".

EI TAE y Focusing ha facilitado la conexión del/la alumno/a con las sensaciones que tenían respecto a una situación o temática y, a su vez, todo ese entramado de conocimiento vivencial sin articular lo han trasladado al espacio simbólico de la palabra: "Entrelazar y crear conceptos nuevos y novedosos ha sido muy enriquecedor, al final encontrar un nexo entre dos conceptos que al principio no tenían nada en común" (A2). Las sensaciones han estado muy presentes durante el proceso: "Cuando hablábamos de sensaciones... me ha ayudado a ponerle nombre a mis experiencias y vivencias $y$ acotar y dar nombre a lo qué exactamente quería hacer en mi TFG" (A2). Eso ha traído que reflexionar haya estado siempre unido con el sentir. Han ido de la mano: "Cuando uno sabe lo que quiere hacer en el TFG...es...que ya puedes discriminar y conectar la información, lo que me vale, y puedes coger ideas nuevas también" (A1).

\section{El alumnado ha llegado a utilizar las herramientas de modo autónomo.}

En ciertos momentos del trabajo varios/as alumnos/as han utilizado los ejercicios fuera de las sesiones de tutoría, es decir, fuera de la supervisión docente: "Abrir y cerrar lo he utilizado en todas las fases. Es más, he cogido un hábito: antes de escribir algo, escribo a mano las ideas que me brotan, para luego ordenarlas..." (A4). El impacto sobre algunos/as alumnos/as es destacable, al transferirlo a procesos autónomos de aprendizaje. "Yo hacía listas desde las sensaciones para el marco teórico, para el 
desarrollo metodológico, para el guión de las entrevistas... y me guiaba por la sensación que me daba la pregunta motriz" (A15).

\section{CONCLUSIONES}

Tras la finalización de este proyecto hemos podido constatar que el conocimiento implícito es una fuente inagotable de creación de nuevos saberes. La introducción del enfoque corporal en el proceso formativo universitario es enriquecedora y ayuda en el transitar desde la incertidumbre resuelta hasta la incertidumbre que queda por resolver (Wagensberg, 2008).

Partir de las sensaciones corporales para el diseño y realización del TFG trae consigo la conexión del alumnado con sus propias vivencias, y desde ahí, la creación de su propio discurso. Por ello, encontramos que es una estrategia válida para trabajar las competencias que persigue el TFG, bien para el desarrollo de una creación propia, bien para profundizar en una temática novedosa, así como para la redacción futura de propuestas profesionales. Es decir, el alumnado puede construir significados desde su experiencia (Gromi, 2013) para así estimular la inteligencia del corazón, como indica Scharmer (2020).

Las herramientas TAE y Focusing utilizadas para concretizar la guía se muestran adecuadas para dar apoyo y respuesta a esa inquietud que emerge desde las vivencias recogidas en diferentes campos de experiencia del ámbito educativo.

La experimentación por parte del alumnado de nuevos espacios de indagación hace que transite al espacio profesional con una nueva mirada y herramientas que le posibilitarán la construcción de nuevas realidades. Para ello, plasmar en una guía herramientas de TAE y Focusing (Gendlin, 1983, 1997; Tokumaru, 2011) es fundamental para el proceso de indagación. Como indican Nagamine et al. (2018) TAE podría ser una opción óptima aportando claridad a las actividades de reflexión para que sean productivas y significativas.

De cara al futuro son varios los nuevos caminos por transitar. Por un lado, es necesaria ampliar la experiencia llevada a cabo más allá de los grados pertenecientes a las ciencias sociales. De esta manera se puede obtener una visión complementaria que tenga por objeto mejorar las herramientas que hemos puesto en práctica que permitiera generar una guía didáctica que respondiera a la totalidad de los grados.

Por otra parte, desde este nuevo lugar que proponemos, se genera un nuevo escenario para la innovación desde donde podrían surgir nuevas propuestas de formación del profesorado universitario que permitiera acompañar al alumnado en las reflexiones sentidas corporalmente. 


\section{REFERENCIAS}

Aguilar, E. (2011). Niveles experienciales en grupo de prácticas de Pensar desde Borde (PDB/TAE) de Gendlin. Miscelánea Comillas. Revista de Ciencias Humanas y Sociales, 69, 134, 55-82.

Alemany, C. (2007). Manual práctico de focusing de Gendlin. Bilbao: Desclée de Brouwer.

Alemany, C., \& Aguilar, E. (2019). Homenaje a Eugene Gendlin, psicoterapeuta, humanista e investigador, con motivo de su muerte. Miscelánea Comillas. Revista de Ciencias Humanas y Sociales, 77, 150, 5-28.

Aramendi, P., Arburua, R.M., \& Buján, K. (2018). El aprendizaje basado en la indagación en la enseñanza secundaria. Revista de Investigación Educativa, 36(1), 109-124.

Barceló, T. (2015). La creación de significado. El modelo de filosofía experiencial del lenguaje de Eugene T. Gendlin. Miscelánea Comillas. Revista de Ciencias Humanas y Sociales, 73, 142, 5-34.

Dewey, J. (2004). Experiencia y Educación. Madrid: Biblioteca Nueva.

Esteves, D. (2018). Colaborar para innovar: contribuciones desde un caso portugués para rediseñar la noción de innovación educativa. Revista Educación, Política y Sociedad, 3 (1), 7-30. https://bit.ly/2wBYw5N

Ferrer, V., Carmona, M., \& Soria, V. (2008). El Trabajo Fin de Grado. Guía para estudiantes, docentes y agentes colaboradores. Madrid: McGraw-Hill.

Freire, P. (2008). Pedagogía del oprimido. Madrid: Siglo XXI.

Gadamer, H.G. (1977). Verdad y método. Salamanca: Sígueme.

Gendlin, E.T. (1983). Focusing: proceso y técnica del enfoque corporal. Bilbao: Mensajero.

Gendlin, E.T. (1997). La escala experiencial. En C. Alemany (coord.), Psicoterapia experiencial y focusing. La aportación de E.T. Gendlin (pp.101-108). Bilbao: Desclée de Brouwer.

Gendlin, E.T. (2009). Introducción a "Pensando desde el borde" (Thinking at the Edge). https://bit.ly/3Ao0Yex

Gromi, A. (2013). Juzgar. De la opinión no fundamentada al juicio elaborado. Madrid: Narcea.

Jordi, R. (2011). Reframing the Concept of Reflection: Consciousness, Experiential Learning, and Reflective Learning Practices. Adult Education Quarterly 61(2), $181-197$.

Krycka, K. (2015). Thinking at the Edge: Where Theory and Practice Meet to Create Fresh Understandings. Indo-Pacific Journal of Phenomenology, 6, 1-10.

Lukas, J.F., \& Santiago, K. (2009). Evaluación educativa. Madrid: Alianza Editorial.

Miles, M.B. \& Huberman, A.M. (1994). Qualitative data analysis: an expanded sourcebook. London: Sage.

Nagamine T., Fujieda Y., \& lida A. (2018). The Role of Emotions in Reflective Teaching in Second Language Classrooms: Felt Sense, Emotionality, and Practical Knowledge Acquisition. En J. Martínez (ed), Emotions in Second Language Teaching.

Peñaherrera, M., Chiluiza, K., \& Ortiz, A. (2014). Inclusión del Aprendizaje Basado en Investigación $(\mathrm{ABI})$ como práctica pedagógica en el diseño de programas de postgrados en Ecuador. Elaboración de una propuesta. Journal for Educators, Teachers and Trainers, 5(2), 204 - 220.

Prigogine, I. (2008). Las leyes del caos. Madrid: Crítica.

Scharmer, O. (2020). How to reinvent the 21st century University. Tackling the lack of vertical literacy. https://bit.ly/2YxNdxf

Sterling, S. (2004). Higher Education, Sustainability, and the role of systemic learning. In P.B. Corcoran \& A.E.J. Wals (eds). Higher Education and the Challenge of Sustainability. https://doi.org/10.1007/0-306-48515-X_5. 
Tokumaru, S. (2011). Qualitative research with TAE steps. Thinking at the Edge: Theory and Aplications. Japón: Keisuisha.

UPV-EHU (2019). Bases para el desarrollo curricular de las titulaciones oficiales de la UPV/EHU. https://bit.ly/3FwiaSY.

Velarde, J. (1996). Pensamiento difuso, pero no confuso: de Aristóteles a Zadeh (y vuelta). Psicothema, 8, 2, 435-466.

Wagensberg, J. (2008). El gozo intelectual. Teoría y práctica sobre la inteligibilidad y la belleza. Barcelona: Tusquets.

Zadeh, L. (2014). What is Fuzzy Logic and What Does it Have to Offer? https://bit.ly/3mH4s78.

Zambrano, M. (1996). Filosofía y Poesía. México: Fondo de Cultura Económica. 